\title{
Safety and Diagnostic Yield of Transbronchial Lung Cryobiopsy in Diffuse Parenchymal Lung Diseases: A Comparative Study versus Video-Assisted Thoracoscopic Lung Biopsy and a Systematic Review of the Literature
}

\author{
Claudia Ravaglia $^{a}$ Martina Bonifazi ${ }^{\mathrm{e}} \mathrm{f} \quad$ Athol U. Wells ${ }^{\mathrm{n}}$ Sara Tomassetti ${ }^{\mathrm{a}}$ Carlo Gurioli $^{\mathrm{a}}$ \\ Sara Piciucchic Alessandra Dubini ${ }^{d}$ Paola Tantalocco $^{a}$ Stefano Sanna ${ }^{b}$ Eva Negrig \\ Irene Tramacere $^{\mathrm{h}}$ Valentina Anna Ventura ${ }^{j} \quad$ Alberto Cavazzak $^{k}$ Andrea Rossi $^{l}$ Marco Chilosi $^{\mathrm{m}}$ \\ Carlo La Vecchiai Stefano Gasparini ${ }^{\mathrm{e}, \mathrm{f}}$ Venerino Poletti ${ }^{\mathrm{a}, \mathrm{o}}$ \\ aPulmonology Unit and ${ }^{\mathrm{b}}$ Thoracic Surgery Unit, Department of Thoracic Diseases, and Departments of ${ }^{\mathrm{C}}$ Radiology \\ and dPathology, G.B. Morgagni - L. Pierantoni Hospital, Forlì, e Pulmonology Unit, Department of Internal \\ Medicine, Azienda Ospedaliero-Universitaria 'Ospedali Riuniti', Foggia, ${ }^{f}$ Department of Biomedical Sciences and \\ Public Health, Università Politecnica delle Marche, Ancona, 9 Department of Epidemiology, 'Mario Negri' Institute \\ for Pharmacological Research-IRCCS, h Neuroepidemiology Units, IRCCS Foundation, 'Carlo Besta' Neurological

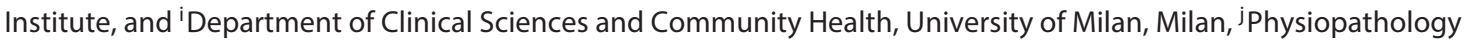

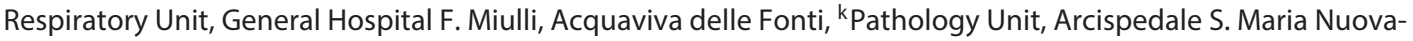 \\ IRCCS, Reggio Emilia, and 'Pulmonary Unit and m Department of Pathology, University of Verona, Verona, Italy; \\ ${ }^{n}$ Interstitial Lung Disease Unit, Royal Brompton Hospital, London, UK; ${ }^{\circ}$ Department of Respiratory Diseases and \\ Allergology, Aarhus University Hospital, Aarhus, Denmark
}

\section{Key Words}

Interstitial lung disease · Cryobiopsy · Video-assisted thoracoscopic surgery

\begin{abstract}
Background: A diagnosis of interstitial lung diseases (ILDs) may include surgical lung biopsy (SLB), which is associated with significant morbidity and mortality and also appreciable costs. Transbronchial lung cryobiopsy (TBLC) is adopting an important role. Objectives: The aim of this study was to compare the diagnostic yield (DY) and safety of TBLC and SLB in a large cohort of patients and to perform a systematic review of the literature as well as a meta-analysis. Methods: We performed a retrospective analysis of 447 cases with ILD undergoing TBLC and/or SLB and a systematic review of the
\end{abstract}

\section{KARGER}

E-Mail karger@karger.com www.karger.com/res literature (MEDLINE and Embase for all original articles on the DY and safety of TBLC in ILDs up to July 2015). Results: A total of 150 patients underwent SLB and 297 underwent TBLC. The median time of hospitalization was 6.1 days (SLB) and 2.6 days (TBLC; $p<0.0001$ ). Mortality due to adverse events was observed for $2.7 \%$ (SLB) and $0.3 \%$ (TBLC) of the patients. Pneumothorax was the most common complication after TBLC (20.2\%). No severe bleeding was observed. TBLC was diagnostic for 246 patients (82.8\%), SLB for 148 patients $(98.7 \%, p=0.013)$. A meta-analysis of 15 investigations including 781 patients revealed an overall DY of 0.81 (0.75-0.87); the overall pooled probability of developing a pneumothorax, as retrieved from 15 studies including 994 patients, was 0.06 (95\% Cl 0.02-0.11). Conclusion: Cryobiopsy is safe and has lower complication and mortality rates compared to SLB. TBLC might, therefore, be considered the
(C) 2016 S. Karger AG Basel

0025-7931/16/0913-0215\$39.50/0
Venerino Poletti, MD

Pulmonology Unit, Department of Thoracic Diseases

G.B. Morgagni - L. Pierantoni Hospital, Via C. Forlanini 34 IT-47100 Forlì (Italy)

E-Mail venerino.poletti@gmail.com 
first diagnostic approach for obtaining tissue in ILDs, reserving the surgical approach for cases in which TBLC is not diagnostic.

(c) 2016 S. Karger AG, Basel

\section{Introduction}

According to current scientific thinking and international guidelines, surgical lung biopsy (SLB) is indicated in diffuse parenchymal lung diseases (DPLDs) when a noninvasive diagnosis cannot confidently be made, with the procedure of choice in today's surgical setting being video-assisted thoracoscopic surgery (VATS) [1, 2]. However, SLB is associated with significant morbidity and mortality as well as appreciable costs [3-6], and many subjects are excluded because of increased risks of perioperative morbidity and mortality (by a combination of advanced age, comorbidity, severe respiratory failure, and pulmonary hypertension). Furthermore, some subjects do not give their consent to a surgical intervention. Cryobiopsy has demonstrated a superior diagnostic yield (DY) in comparison to conventional forceps biopsy [710], but only few studies have compared its complications and DY with those of SLB.

Our aim was to retrospectively assess and compare the DY and safety of transbronchial lung cryobiopsy (TBLC) and SLB in a large cohort of patients from clinical practice. Moreover, we performed a systematic review of the literature and a meta-analysis of the data from all the available studies in order to provide an overall estimate of the DY and safety of this procedure.

\section{Materials and Methods}

In this retrospective analysis of a prospective clinical protocol, we analyzed all the patients with interstitial lung disease (ILD) undergoing TBLC from March 2011 through March 2015 and/or SLB from January 2003 through March 2015 because a diagnosis could not be achieved noninvasively according to current international consensus criteria $[11,12]$. The study was conducted at the Pulmonology Unit of G.B. Morgagni - L. Pierantoni Hospital in Forlì (Italy) and submitted to the Ethics Committee of IRST IRCCS Area Vasta Romagna. Subjects were identified by a retrospective chart review.

At the time of the procedure, we collected data regarding age, gender, past medical history, medications, smoking history, environmental exposure history, physical examination findings, laboratory results, and pulmonary function. High-resolution computed tomography (HRCT) images performed within 1 month prior to the procedure were reviewed by an expert radiologist (S.P.). One- or $1.5-\mathrm{mm}$ collimation sections were obtained at $10-\mathrm{mm}$ intervals, or volumetrically on multidetector CT scanners with 0.6- or 1-mm collimation and 1-mm reconstruction; all images were reviewed at window settings optimized for lung parenchyma (width: 1,500-1,600 HU; level: 2,500-2,600 HU). All patients included in this study had an HRCT pattern of ILD defined as 'possible usual interstitial pneumonia' (UIP) or 'inconsistent with UIP', according to current guidelines [12], and also inconsistent with sarcoidosis, Langerhans cell histiocytosis, or other patterns of cystic or nodular diseases.

Cryobiopsy was performed as previously described [10]: patients were deeply sedated with intravenous propofol and remifentanil and intubated with a rigid tracheoscope; oxygen was administered continuously, and spontaneous breathing was maintained during the procedure. A flexible cryoprobe measuring $90 \mathrm{~cm}$ in length and $2.4 \mathrm{~mm}$ in diameter was used (ERBE, Germany), and the biopsies were obtained under fluoroscopic guidance using the flexible bronchoscope, which was inserted through the rigid tube into the selected bronchus; once brought into position, the probe was cooled for approximately $5 \mathrm{~s}$, after which it was retracted, with the frozen lung tissue being attached to the probe's tip. A distance $<10 \mathrm{~mm}$ from the thoracic wall, or from what was considered to be the pleura due to the resistance found by the pulmonologist, was considered optimal. A Fogarty balloon was prophylactically placed in the lobar bronchus near the biopsy segment and routinely inflated after sampling to minimize the consequence of hemorrhage. A bronchoalveolar lavage (BAL) was performed in all patients during the same bronchoscopy as the transbronchial cryobiopsies. BAL was performed with sequential infusions of six 25 - $\mathrm{ml}$ aliquots of $0.9 \%$ saline solution; the first 2 aliquots were used for microbiological investigations, and the fluid retrieved from the successive aliquots was pooled and divided for different analyses (cell count and cytological and cytofluorimetric analysis). BAL had also been performed in all patients of the VATS group. VATS was carried out under general anesthesia, utilizing double-lumen endobronchial intubation and single-lung ventilation. The standard 3-port VATS technique was used. The choice of the site and side of biopsy was guided by CT imaging. A single $32-\mathrm{Fr}$ chest drain was inserted into one of the anterior port sites, with the other two port sites being closed with sutures.

Hematoxylin-eosin-stained slides from biopsies were sent to three pathologists (A.D. and V.P. from our hospital and A.C. from a different hospital). Before the multidisciplinary meeting, the three pathologists examined the pathologic specimens independently and then discussed the cases and reached a final, common pathology interpretation of each case.

At least one fragment of alveolated lung parenchyma was required to classify the biopsy as adequate. The samples were classified as providing a diagnostic pattern or not ('nondiagnostic pattern'). The pathologic diagnosis of UIP was made according to current guideline criteria [12].

All cases that exhibited pathologic findings suggestive of, or definitive for, an alternative diagnosis were considered non-UIP and classified according to the characteristic pathologic features, i.e. nonspecific interstitial pneumonia, desquamative interstitial pneumonia or respiratory bronchiolitis-ILD, organizing pneumonia, diffuse alveolar damage, hypersensitivity pneumonitis, sarcoidosis and other granulomatosis, other, or nondiagnostic. All cases $(100 \%)$ were subsequently discussed in the multidisciplinary meeting.

Adverse events included pneumothorax, severe bleeding, persistent fever, prolonged air leak, acute exacerbation, transient re- 
Table 1. Patient characteristics

\begin{tabular}{|c|c|c|c|}
\hline & $\begin{array}{l}\text { SLB (VATS) group } \\
(\mathrm{n}=150)\end{array}$ & $\begin{array}{l}\text { TBLC group } \\
(\mathrm{n}=297)\end{array}$ & $\mathrm{p}$ value \\
\hline Patients & 150 & 297 & \\
\hline Age, years & $59(15-74)$ & $60(21-78)$ & 0.278 \\
\hline \multicolumn{4}{|l|}{ Gender } \\
\hline Male & $85(56.7)$ & $172(57.9)$ & 0.801 \\
\hline Female & $65(43.3)$ & $125(42.1)$ & \\
\hline \multicolumn{4}{|l|}{ Smoking } \\
\hline Current smoker & $21(14.0)$ & $40(13.5)$ & \\
\hline Former smoker & $63(42.0)$ & $124(41.8)$ & \\
\hline Nonsmoker & $65(43.3)$ & $133(44.8)$ & 0.968 \\
\hline FVC, \% predicted & $80.0(20.0-136.0)$ & $86.0(37.0-137.0)$ & 0.072 \\
\hline $\mathrm{FEV}_{1}, \%$ predicted & $83.0(33.0-133.0)$ & $88.0(36.0-144.0)$ & 0.034 \\
\hline DLCO, \% predicted & $57.0(19.0-122.0)$ & $58.8(14.0-121.0)$ & 0.078 \\
\hline Tiffeneau index & $86(62.0-105.0)$ & $86(60.0-124.0)$ & 0.85 \\
\hline \multicolumn{4}{|l|}{ Histological pattern } \\
\hline DIP/RB-ILD & $11(7.3)$ & $12(4.0)$ & \\
\hline UIP & $74(49.3)$ & $92(31.0)$ & \\
\hline NSIP & $23(15.3)$ & $25(8.4)$ & \\
\hline $\mathrm{DAD}$ & $1(0.7)$ & $4(1.3)$ & \\
\hline $\mathrm{OP}$ & $8(5.3)$ & $31(10.4)$ & \\
\hline HP & $7(4.7)$ & $24(8.1)$ & \\
\hline SAR & $8(5.3)$ & $22(7.4)$ & \\
\hline $\begin{array}{l}\text { Other (neoplasms, eosinophilic pneumonia, } \\
\text { follicular bronchiolitis, alveolar proteinosis, } \\
\text { vasculitis, AFOP, DIPNECH, or PLCH) }\end{array}$ & $16(10.7)$ & $36(12.1)$ & \\
\hline Nondiagnostic pattern & $2(1.3)$ & $51(17.2)$ & 0.013 \\
\hline
\end{tabular}

Values are expressed as $\mathrm{n}(\%)$ or medians (ranges). Italics denote significance. DIP $=$ Desquamative interstitial pneumonia; RB-ILD = respiratory bronchiolitis-ILD; NSIP = nonspecific interstitial pneumonia; DAD = diffuse alveolar damage; $\mathrm{OP}=$ organizing pneumonia; $\mathrm{HP}=$ hypersensitivity pneumonitis; $\mathrm{SAR}=$ sarcoidosis and other granulomatosis; AFOP = acute fibrinous and organizing pneumonia; DIPNECH = diffuse idiopathic pulmonary neuroendocrine cell hyperplasia; PLCH = pulmonary Langerhans cell histiocytosis.

spiratory failure, pneumonia/empyema, and other events. Severe bleeding (grade 3) was defined as 'causing hemodynamic or respiratory instability, requiring tamponade or other surgical interventions, transfusions, or admission to the intensive care unit'; we did not include mild-to-moderate bleeding of grade 0 (no bleeding), grade 1 (bleeding requiring suction to clear but no other endoscopic procedures), or grade 2 (bleeding requiring endoscopic procedures such as bronchial occlusion-collapse and/or instillation of ice-cold saline) [13]. A prolonged air leak was defined as an air leak lasting $\geq 5$ days after the procedure $[14,15]$.

The systematic review of the literature was performed according to the guidelines developed by the Meta-Analysis of Observational Studies in Epidemiology (MOOSE) group [16]. We searched MEDLINE and Embase for all original articles on the DY and safety of transbronchial cryobiopsy for the diagnosis of DPLDs published up to July 2015, using a combination of free text and MeSH/ EMTREE terms related to DPLDs and cryobiopsy technique. The electronic search was supplemented with gray literature and by hand-searching the bibliographies of relevant articles. The following criteria were established for inclusion: (1) studies reporting data on the DY and safety of transbronchial cryobiopsy for the diagnosis of DPLDs (i.e. suspected ILDs or pulmonary infiltrates) and (2) studies reporting data on the safety of transbronchial cryobiopsy in routine surveillance after lung transplantation or for research purposes. Exclusion criteria were: (1) studies reporting data on the DY and safety of transbronchial cryobiopsy for the diagnosis of peripheral lung lesions; (2) studies reporting data on the DY and safety of transbronchial cryobiopsy for the diagnosis of central lung lesions, and (3) studies reporting data on the DY of transbronchial cryobiopsy in routine surveillance after lung transplantation. Two independent authors (M.B. and C.R.) reviewed all titles/abstracts and retrieved detailed full texts of potentially relevant articles. Disagreements were resolved by discussion. When multiple reports were available on the same cohort of patients, we included the most recent or informative one. The two reviewers independently retrieved information on the country, study design, number of subjects, study population, main outcomes, procedural aspects, number and size of samples, prevalence of (or high probability of/high confidence about) the UIP pattern at histology, DY, and safety. The measure of interest for the DY and safety analyses 
Table 2. Safety profile results for SLB (VATS) and TBLC

\begin{tabular}{|c|c|c|c|}
\hline & $\begin{array}{l}\text { SLB (VATS) } \\
(\mathrm{n}=150)\end{array}$ & $\begin{array}{l}\text { TBLC } \\
(\mathrm{n}=297)\end{array}$ & $\mathrm{p}$ value \\
\hline Days of hospitalization & $6.1(3-48)$ & $2.6(0-17)$ & $<0.0001$ \\
\hline \multicolumn{4}{|l|}{ Adverse events } \\
\hline Severe bleeding & $0(0.0)$ & $0(0.0)$ & \\
\hline Persistent fever & $7(4.7)$ & $0(0.0)$ & \\
\hline Prolonged air leak & $5(3.3)$ & $1(0.3)$ & \\
\hline Acute exacerbation & $5(3.3)$ & $1(0.3)$ & \\
\hline Pneumonia/empyema & $3(2.0)$ & $0(0.0)$ & \\
\hline Transient respiratory failure & $0(0.0)$ & $2(0.7)$ & \\
\hline Miscellanea & $0(0.0)$ & $2(0.7)$ & \\
\hline Pneumothorax (in total) & NA (NA) & $60(20.20)$ & \\
\hline Pneumothorax with drainage & NA (NA) & $46(15.50)$ & \\
\hline Days of drainage & $3.75(2-40)$ & $4.65(2-15)$ & 0.138 \\
\hline Patients with 0 adverse events & $131(87.3)$ & $220(74.1)$ & \\
\hline Patients with 1 adverse event & $16(10.7)$ & $75(25.3)$ & \\
\hline Patients with 2 adverse events & $3(2.0)$ & $1(0.3)$ & \\
\hline Patients with 3 adverse events & $0(0.0)$ & $1(0.3)$ & \\
\hline Time to 1st adverse event after biopsy, days & $27.5 \pm 73.9$ & $0.6 \pm 2.0$ & $<0.0001$ \\
\hline \multirow[t]{2}{*}{ Mortality due to adverse event } & $4 / 150(2.7)$ & $1 / 297(0.3)$ & 0.045 \\
\hline & $4 / 20(20.0)$ & $1 / 66(1.5)$ & 0.01 \\
\hline \multicolumn{4}{|l|}{ Survival } \\
\hline Alive & $88(58.7)$ & $272(91.6)$ & \\
\hline Dead & $43(28.7)$ & $13(4.4)$ & \\
\hline Transplantation & $4(2.7)$ & $1(0.3)$ & \\
\hline
\end{tabular}

Values are expressed as $\mathrm{n}(\%)$, mean (range), or mean \pm SD. Italics denote significance. NA = Not applicable.

was the proportion of events. In order to obtain pooled estimates, we performed meta-analyses of proportions using the FreemanTukey double arcsine transformation method [17] and generated the corresponding forest plots. Heterogeneity between studies was assessed using the $\chi^{2}$ test [18], defining a significant heterogeneity as a $\mathrm{p}$ value $<0.10$, while inconsistency was quantified using the $\mathrm{I}^{2}$ statistic.

\section{Results}

We collected data on 150 cases in the VATS group and 297 cases in the TBLC group; 14 patients with cryobiopsy subsequently also underwent an SLB due to nondiagnostic samples, and 1 patient subsequently had a cryobiopsy repeated for the same reason. The baseline characteristics of each group were similar (table 1). In the SLB group, the pathologists identified histopathologic criteria sufficient to define a characteristic pattern in 148 patients (98.7\%); in the TBLC group, a characteristic pattern was obtained in 246 cases $(82.8 \%$; $\mathrm{p}=0.013)$.

Safety outcomes are summarized in table 2 . The median time of hospitalization was 6.1 days after SLB and 2.6 days after TBLC ( $\mathrm{p}<0.0001) ; 16$ patients were discharged home on the day of the procedure. The time to the first adverse event after biopsy was 27.5 days for VATS versus 0.6 days for cryobiopsy.

Mortality due to adverse events after SLB was observed in 4 patients (2.7\% of the total), and $20 \%$ of the adverse events $(4 / 20)$ were fatal; death occurred within 60 days and was caused by acute exacerbation of idiopathic pulmonary fibrosis (IPF) in all cases. Prolonged air leaks were treated with a prolonged chest tube drainage in $3 / 5$ patients; the remaining 2 patients were treated with a blood patch, and in 1 case also with surgical revision. In the TBLC group, only 1 patient died after 7 days $(0.3 \%$ of the total) with acute exacerbation of IPF (coexistence of diffuse alveolar damage and a UIP pattern at autopsy) following massive pneumothorax (treated with drainage and high-flow oxygen) and a prolonged air leak. Pneumothorax was the most common complication after cryobiopsy, occurring in 60 patients $(20.2 \%), 46$ cases (15.5\% of the total) requiring drainage. No patients needed an intervention to control bleeding, and there were no cases with persistent fever or pneumonia/empyema. Other complications were: tran- 


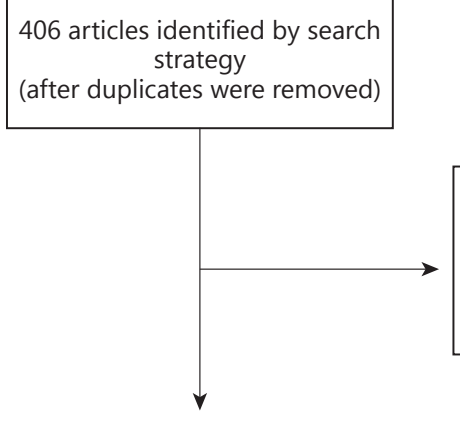

369 articles excluded after screening of title/abstract because they were not relevant

(i.e. animal studies, studies not reporting outcomes

of interest, studies on cryobiopsies in other

populations, reviews, case reports)

37 potentially relevant studies

retrieved for detailed (full-text) evaluation

Fig. 1. Flowchart of study selection.

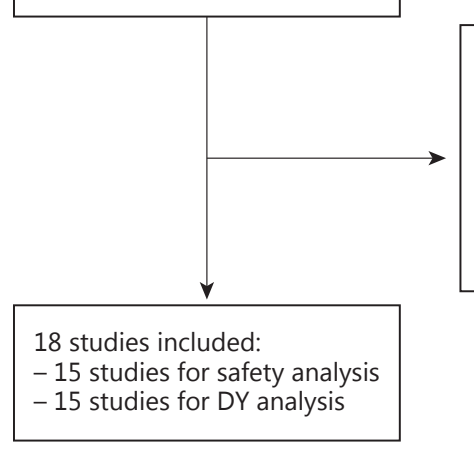

19 studies excluded:

-3 case reports

-4 review articles

-2 studies not reporting outcomes of interest

- 10 studies reporting outcomes of interest for

cryobiopsies in other populations (i.e. patients

with central lesions or peripheral pulmonary nodules)

sient respiratory failure (2 patients; $0.7 \%$ ) and neurologic manifestations (seizures in 2 patients; $0.7 \%$ ). In the VATS group, lung function was more compromised in patients who developed subsequent complications (FVC $70.2 \pm$ 22.0 vs. $82.0 \pm 21.2 \% ; \mathrm{p}=0.029$ ), while in the cryobiopsy group, lung function was found not to be related to the occurrence of these complications. As all patients had undergone BAL with the same procedure and aliquots, it was not possible to find any statistically significant correlation between BAL and side effects in either group.

\section{Review of the Literature}

After removing duplicates between MEDLINE and Embase, the systematic review identified 406 references (fig. 1). The initial screening, based on titles/abstracts, led to the exclusion of 369 papers because they were not relevant (i.e. case reports, review articles); the remaining 37 articles were retrieved for detailed full-text evaluation. Eighteen original studies were included in the present review, and their main characteristics are presented in table 3 [7-9, 19-32]: 15 studies reported data on both DY and safety, 2 on safety only, and 1 on DY only.
The summary estimate of DY was provided overall, according to the criteria used for defining diagnostic samples (i.e. either the identification of a specified histological pattern or the final multidisciplinary diagnosis) (fig. 2), and according to selected procedural aspects. The overall DY, derived from 15 investigations including 781 patients, was $0.81(0.75-0.87)$, and a significant heterogeneity between studies was detected $\left(\mathrm{I}^{2} 77.76 \%\right.$; $\left.\mathrm{p}<0.001\right)$. As 2 studies [21,32] provided two different DYs according to the two different diagnostic definitions, we decided to include both values separately in the pooled estimate. However, the sensitivity analysis including only the DY derived from the final histological pattern did not change the overall result (data not shown). The pooled estimates by diagnostic definitions were 0.81 (95\% CI 0.66-0.92; $\mathrm{I}^{2}$ $87.41 \%$; $<$ 0.001) from studies considering multidisciplinary discussion as the final diagnosis ( 7 studies including 300 patients), 0.80 (95\% CI 0.72-0.87; $\mathrm{I}^{2} 68.10 \%$; $\mathrm{p}<$ 0.001 ) from studies referring to the detection of specific histological patterns (8 studies including 564 patients), and 0.90 (95\% CI 0.76-0.99; $\mathrm{I}^{2}$ 95.40\%; $\left.\mathrm{p}<0.001\right)$ from the 2 studies that did not specify their diagnostic criteria 
Table 3. Main characteristics of the original studies included in the systematic review

\begin{tabular}{|c|c|c|c|c|c|c|c|c|c|}
\hline Study & Country & $\begin{array}{l}\text { Study } \\
\text { design }\end{array}$ & Population & $\mathrm{n}$ & Outcomes & Procedural aspects & $\begin{array}{l}\text { Samples, } \\
\text { n/average }\end{array}$ & $\begin{array}{l}\text { Mean area, } \\
\mathrm{mm}^{2} / \\
\text { diameter, } \mathrm{mm}\end{array}$ & $\begin{array}{l}\text { UIP } \\
\text { pattern, } \\
\mathrm{n}(\%)\end{array}$ \\
\hline Kropski [20], 2013 & USA & RCS & $\begin{array}{l}\text { Suspected ILD/ } \\
\text { asymptomatic family } \\
\text { members at risk }\end{array}$ & 37 & DY, safety & ET, conscious sedation & 2 & $64.2 / 8.7 /-$ & $7(28)$ \\
\hline Griff [22], 2011 & Germany & PCS & $\begin{array}{l}\text { Suspected ILD/ } \\
\text { infiltrations }\end{array}$ & 15 & Safety & Conscious sedation & 1 & $17.1 \pm 10.7$ & NA \\
\hline Griff [23], 2014 & Germany & RCS & Suspected ILD & 52 & DY, safety & Conscious sedation & 2 & $-/ 6.9 \pm 4.4$ & $13(25)$ \\
\hline Pajares [9], 2010 & Spain & PCS & Suspected ILD & 10 & DY, safety & FB, sedation & 2 & $9.5 / 3.1$ & 0 \\
\hline $\begin{array}{l}\text { Hernández-González } \\
\text { [24], } 2015\end{array}$ & Spain & RCS & Suspected ILD & 33 & DY, safety & $\begin{array}{l}\text { Conscious sedation, } \\
\text { Fogarty balloon }\end{array}$ & $\geq 3 / 2.7$ & $4 /-/-$ & $6(18.2)$ \\
\hline Gershman [27], 2015 & Israel & RCS & $\begin{array}{l}\text { Suspected ILD/ } \\
\text { immunocompromised } \\
\text { patients with infiltrates/ } \\
\text { lung transplanted }\end{array}$ & 300 & Safety & Conscious sedation & $2-4$ & NA & NA \\
\hline Martín [28], 2014 & Spain & RCS & Suspected ILD & 36 & DY, safety & ET & $-/ 2.7 \pm 0.8$ & NA & $9(25)$ \\
\hline Yarmus [8], 2013 & USA & PCS & Lung transplanted & 17 & Safety & $\begin{array}{l}\text { RB, deep sedation with } \\
\text { jet ventilation }\end{array}$ & 5 & NA & NA \\
\hline Ravaglia [this study] & Italy & PCS & Suspected ILD & 297 & DY, safety & $\mathrm{RB}$, deep sedation & $1-8 /-$ & 44.4/NA & $92(31)$ \\
\hline $\begin{array}{l}\text { Pourabdollah [29], } \\
2014\end{array}$ & Iran & PCS & Suspected ILD & 41 & DY & NA & 1 & $22 / \mathrm{NA}$ & 0 \\
\hline Oezkan [30], 2015 & Germany & RCS & Suspected ILD & 17 & DY, safety & $\mathrm{RB}$ & NA & NA & NA \\
\hline Fruchter [32], 2014 & Israel & RCS & Suspected ILD & 75 & DY, safety & Conscious sedation & $2-4 / 3$ & 9/NA & $7(9.3)$ \\
\hline Fruchter [31], 2013 & Israel & RCS & $\begin{array}{l}\text { Immunocompromised } \\
\text { patients with infiltrates }\end{array}$ & 15 & DY, safety & Conscious sedation & $\mathrm{NA} / 2$ & 9/NA & - \\
\hline
\end{tabular}

RCS = Randomized controlled study; RCT = randomized controlled trial; PCS = prospective case study; CS = controlled study; RB = rigid bronchoscopy; $\mathrm{ET}=$ endotracheal tube; $\mathrm{FB}=$ flexible bronchoscopy; $\mathrm{NA}=$ not available.

(fig. 2). The pooled DYs by procedural aspects, in terms of type of sedation and airway control, were 0.81 (95\% CI 0.76-0.86) from studies with patients undergoing the procedure intubated under deep sedation (11 studies including 625 patients) and 0.83 (95\% CI 0.64-0.97) from studies with patients not intubated under conscious sedation (3 studies including 142 patients) (fig. 3).

With reference to complications, data were retrieved from 15 studies including 994 patients. Overall, 100 pneumothoraces (10\%), 4 transient respiratory failures $(0.4 \%)$, 2 episodes of convulsions ( $0.2 \%), 1$ death $(0.1 \%), 1$ acute exacerbation $(0.1 \%)$, and 1 prolonged air leak $(0.1 \%)$ were reported. Out of the 100 pneumothoraces, 70 required chest tube drainage. The overall pooled probability of developing a pneumothorax was 0.06 (95\% CI 0.02-0.11), while the probability of developing a pneumothorax requiring chest tube drainage was 0.03 (95\% CI 0.01-0.08) (fig. 4). The pooled proportions of pneumothorax according to procedural aspects, in terms of type of sedation and airway control, were 0.07 (95\% CI 0.02-0.14) from studies with patients undergoing the procedure intubated under deep sedation (11 studies including 613 patients) and 0.01 (95\% CI 0.00-0.06) from studies with patients not intubated under conscious sedation ( 3 studies including 367 patients) (fig. 5).

No episodes of severe bleeding (as defined above) were reported. The overall pooled probability of developing a moderate bleeding, as derived from 12 studies including 383 patients, was 0.12 (95\% CI 0.02-0.25) (fig. 6). We did not include our cohort study in the analysis, as the routine preventive use of a Fogarty balloon was considered a major procedural difference. No severe episodes (as defined above) were reported. 


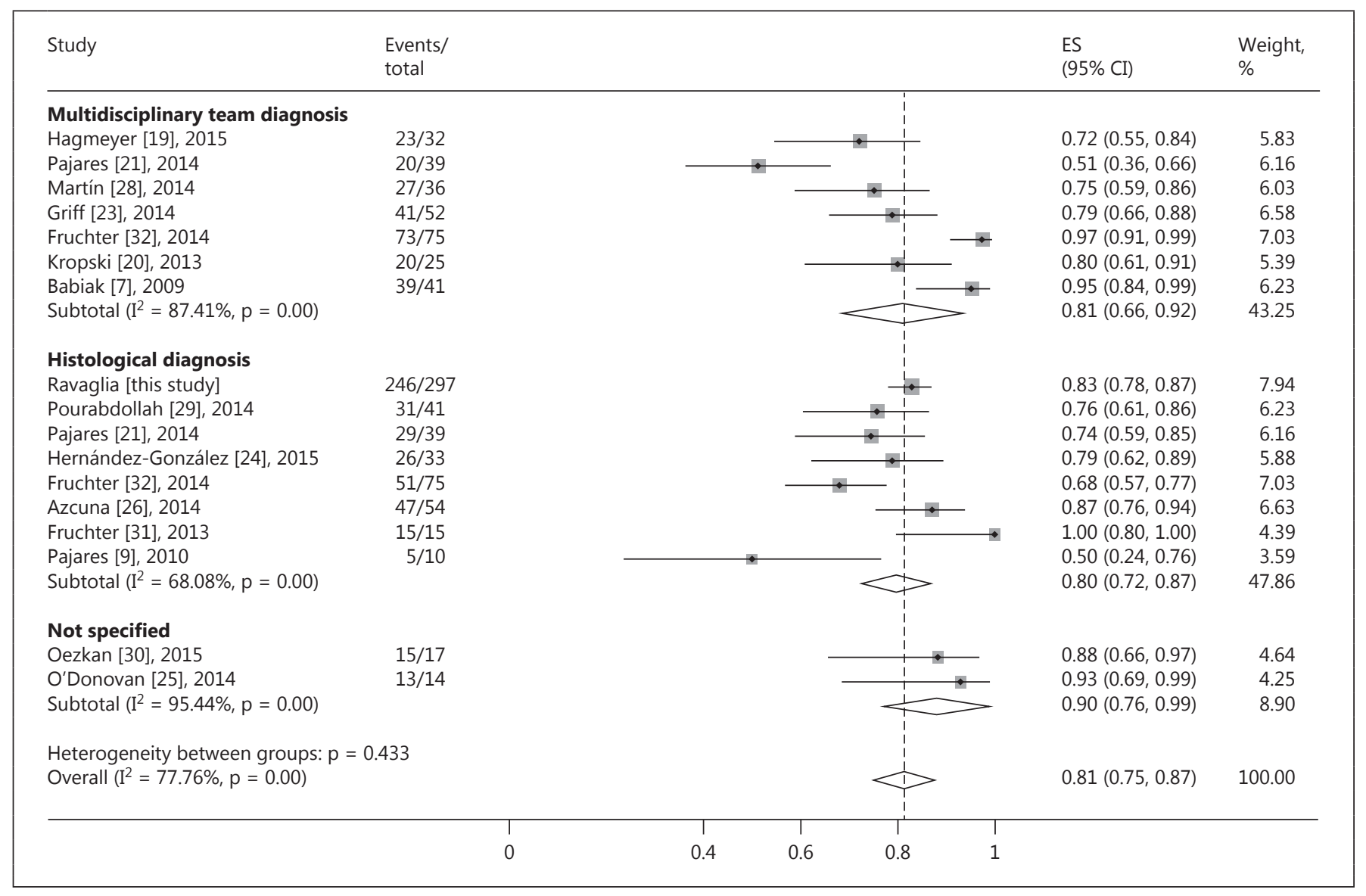

Fig. 2. DY overall and according to diagnostic criteria. ES = Effect size.

\section{Discussion}

Diagnosis of DPLDs is a dynamic multidisciplinary process, requiring close communication between clinicians, radiologists, and pathologists $[33,34]$. SLB is recommended in a large patient subgroup for diagnostic confirmation, in particular in the diagnosis of IPF, in the absence of a UIP pattern on HRCT [2]. However, the procedure requires endotracheal intubation, general anesthesia, chest tube placement, and, typically, hospitalization for several days. Mortality can be observed in 2-6\% at 90 days [3-6], and there is some risk of persistent pain at 12 months $[35,36]$, prolonged air leak, and/or bronchopleural fistula (3-12\%) requiring prolonged hospitalization and/or reoperation [5,37]. Moreover, patients who are ultimately diagnosed with IPF subsequently can experience worse postprocedural outcomes than those with other ILDs $[4-6,38]$, mostly in terms of acute exacerbations [39, 40]; 30-day mortality can increase to
$18.8 \%$ [5]. These data suggest that when IPF is suspected, the benefits of diagnostic confirmation must be weighed against the potential risks of life-threatening complications [41]; in clinical practice, only $10-20 \%$ of fibrotic ILD patients undergo an SLB $[42,43]$.

The advent of TBLC has occurred over the previous decade, and it might soon be integrated in the routine diagnostic pathways $[10,41]$. Current data have shown that this technique appears to be safer than SLB; however, its risks and benefits over VATS have yet to be completely elucidated. The primary objective of our study was to assess the DY and safety of TBLC in a large cohort of patients and to retrospectively compare these outcomes to those obtained from VATS in the diagnostic workup of DPLDs. As expected, the DY of SLB was slightly higher than that of TBLC, but the latter procedure offered significant advantages in terms of safety. Patients with DPLDs who underwent a TBLC had a significantly lower mortality rate following the procedure as compared to the 


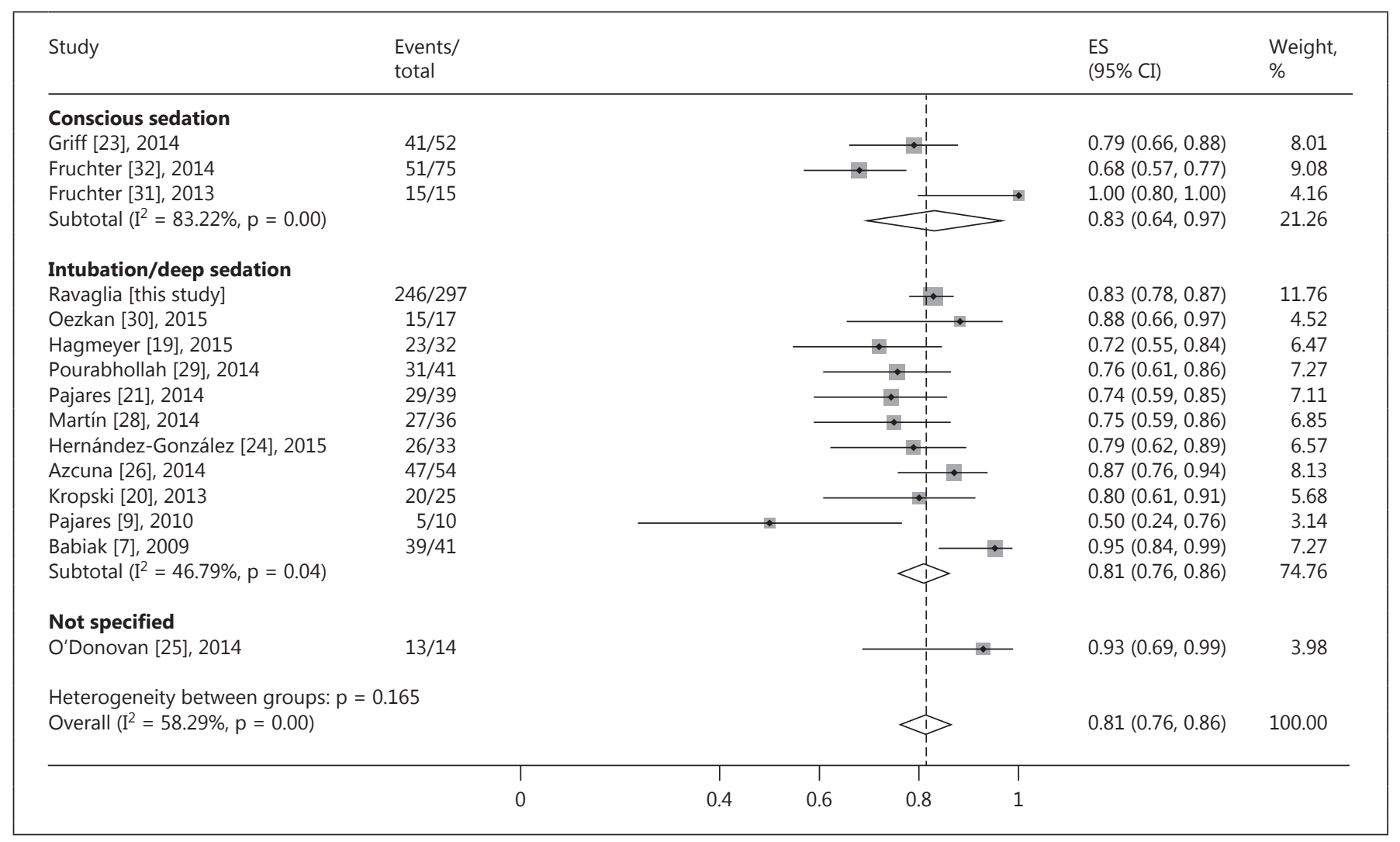

Fig. 3. DY by sedation/intubation. ES = Effect size.

patients undergoing VATS, and the overall mortality rate with this procedure among approximately 1,000 patients was at the very least negligible $(0.1 \%)$. Our results are inconclusive with regard to BAL, maybe due to the selection of retrospective cases that underwent lung biopsy, thus including cases in which BAL was not diagnostic. However, it has recently been demonstrated that the addition of BAL to the clinicoradiological picture of patients with suspected ILD can change the diagnosis only in $8 \%$ of cases [44].

Regarding the length of hospitalization, the reported median time of hospitalization after the SLB procedure for any cause ranges between 4 and 8 days $[43,45]$, with elderly patients with ILDs being at higher risk of prolonged hospitalization [46]. Our data confirm that the time of hospitalization for cryobiopsy is usually shorter than the length of stay after SLB and could also be performed in an outpatient setting. On the other hand, it is striking that the time to the first adverse event after biopsy is much shorter with cryobiopsy than with VATS. This time to adverse events cannot be considered a re- porting bias, as all patients of both groups had been seen at our center after biopsy, in particular patients undergoing cryobiopsy (even if they had been discharged earlier). The time of follow-up after cryobiopsy was 20.99 months, whereas that after VATS was 54.72 months.

In the Introduction, we also mentioned costs (SLB is associated with significant morbidity and mortality and also appreciable costs) [3-6]. Hernández-González et al. [24] demonstrated that systematic cryobiopsies could save between EUR 953.09 and 1,925.29 per patient in comparison to VATS in Spain.

Among the adverse events, pneumothorax represented the most important complication of TBLC, requiring chest tube drainage in 46 cases ( $15.50 \%$ ); however, patient discomfort from incisional and/or chest tube site pain could be avoided, performing cryobiopsy instead of VATS, in $84.5 \%$ of the patients. When chest drainage was necessary, the time of drainage was similar in the two groups. The data on pneumothorax as a major complication were confirmed by the systematic review of the literature on safety profiles (100 out of 109 events). The het- 


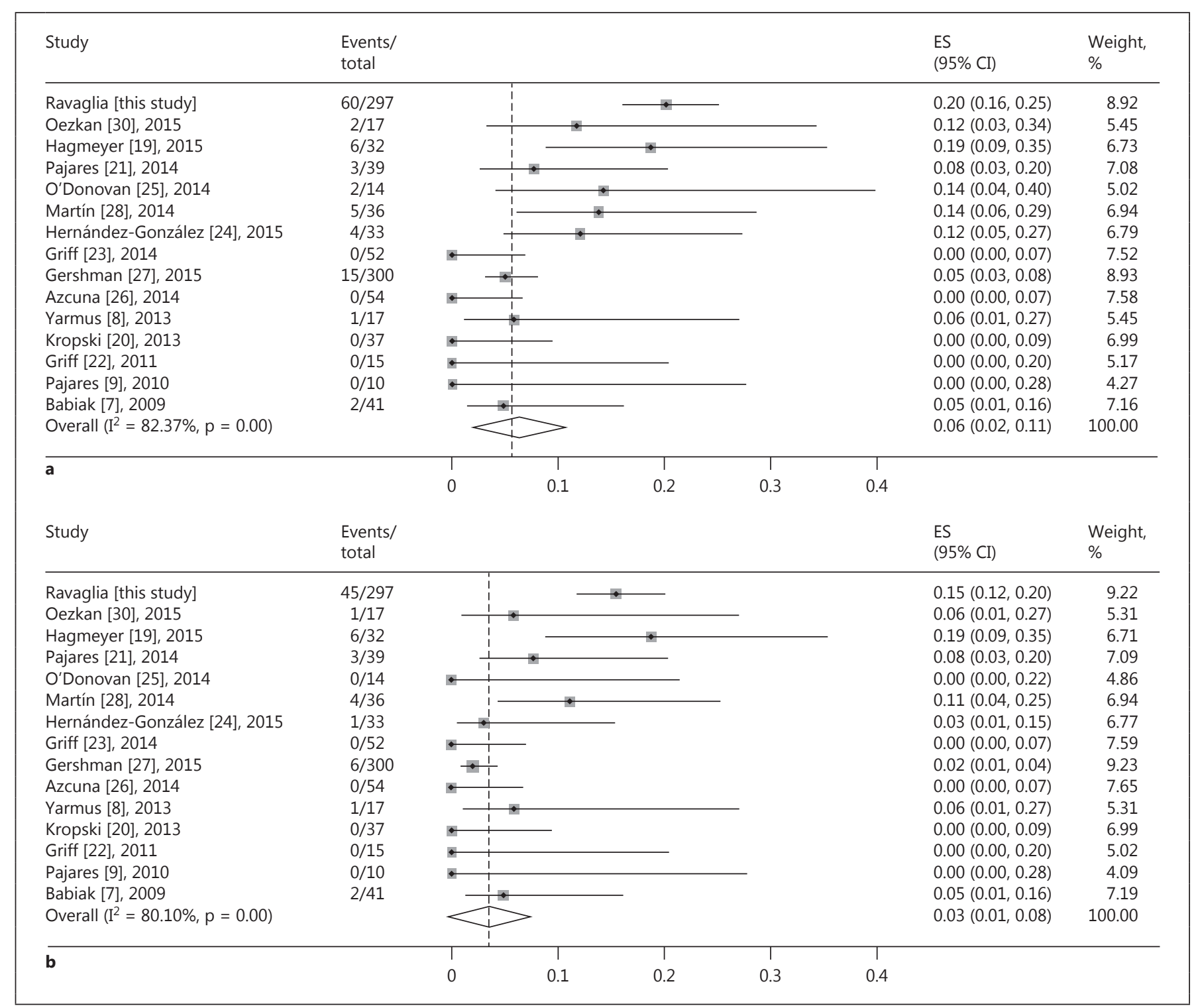

Fig. 4. Pneumothorax overall (a) and requiring a chest tube (b). ES = Effect size.

erogeneity between results was explored via subgroup analysis by procedural aspects; it showed a higher proportion of events among intubated patients undergoing the procedure under deep sedation compared to those under conscious sedation. This could partly be due to the differential prevalence of the UIP pattern between the subgroups, i.e. $25.5 \%$ in the first group and $14.4 \%$ in the second group, and partly due to the lower mean size of samples of patients under conscious sedation. Moreover, in our previous pilot study, we found that the risk of pneumothorax can increase when fragments of pleura are present in the biopsy [10]. However, it was not possible to assess this in the meta-analysis, since data on pleural fragments in the samples were not available in the majority of studies included. At our center, the HRCT fibrosis score of the lower lung zones is significantly increased in patients with pneumothorax compared to those without pneumothorax; furthermore, the higher incidence of pneumothorax seems to correlate significantly with a histological UIP pattern and the bronchoscopist's learning curve [unpubl. data]. 


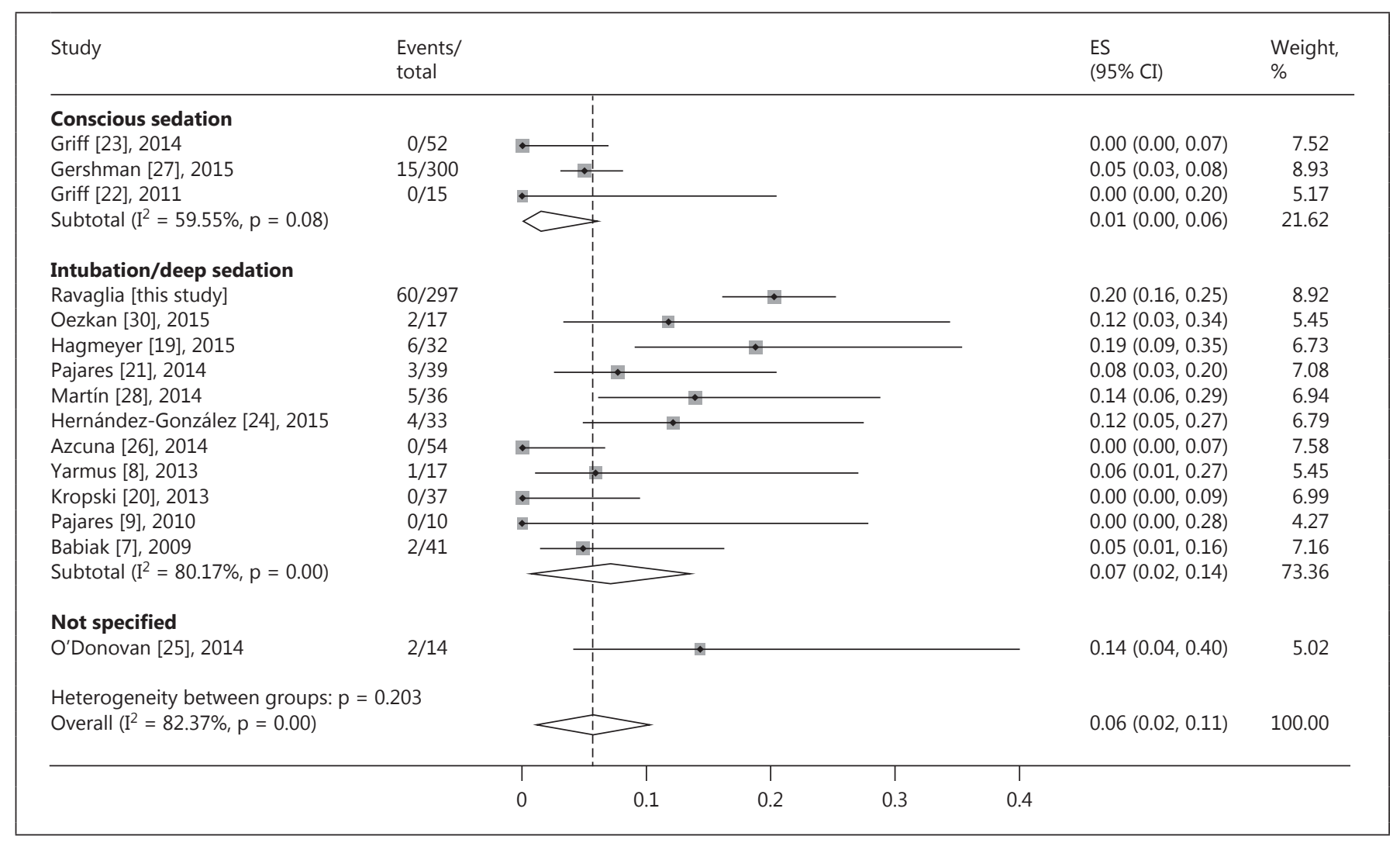

Fig. 5. Pneumothorax by sedation/intubation. ES = Effect size.

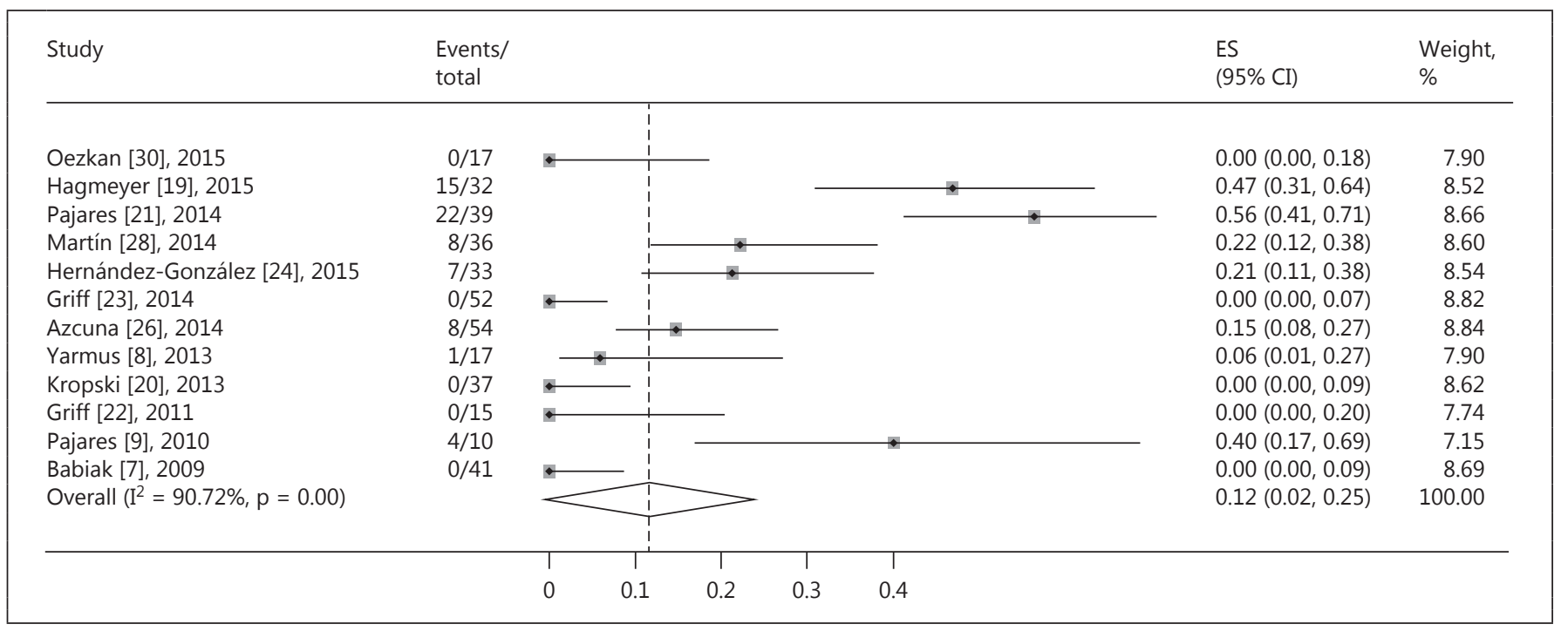

Fig. 6. Moderate bleeding. ES = Effect size. 


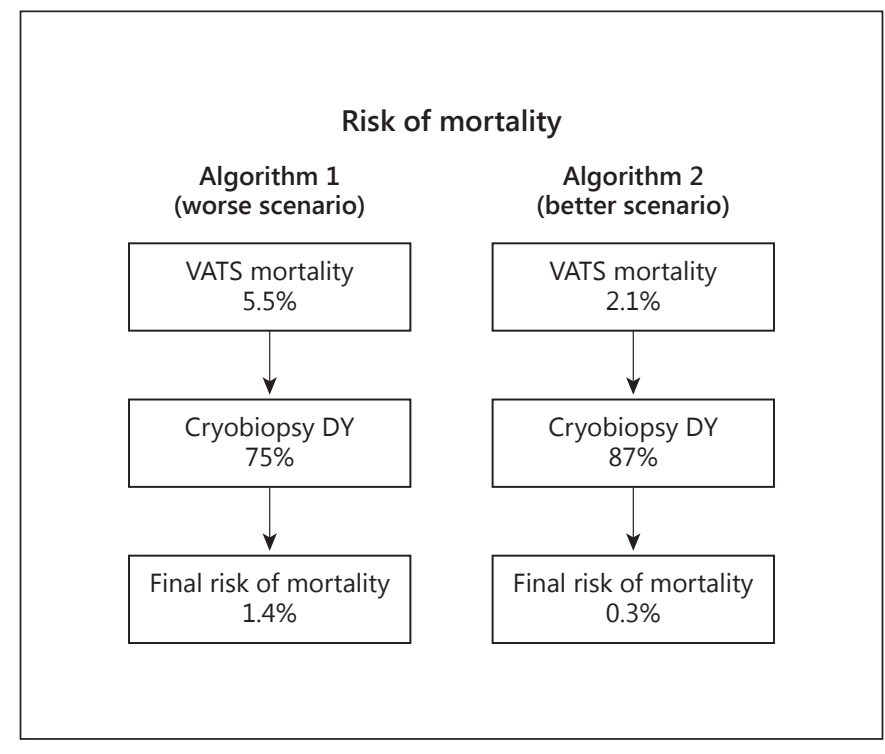

Fig. 7. The 'final' risk of mortality, considering TBLC and VATS together, can vary from $0.3 \%$ (better scenario) to $1.4 \%$ (worse scenario).

No grade 2 and 3 bleeding occurred in our cohort, partly due to the preventive use of a Fogarty balloon, which is inflated immediately while the bronchoscope is being withdrawn from the airway to remove the biopsy specimen from the cryoprobe. Moreover, the hemostatic effects of cooling could probably have contributed to the low incidence of significant bleeding. Data from the systematic review again confirmed this result, since no severe bleeding was reported even in studies not employing the Fogarty balloon. However, a number of moderate bleedings were documented in 7 out of 12 studies reporting on this issue. The pooled estimate of 0.12 , presented in figure 6 , should be interpreted with caution, as the definition of 'moderate' bleeding was vastly different between the studies, making a reliable summary of the data difficult to accomplish. Moreover, the largest cohort studies were not included in this analysis, since Gershman et al. [27] did not distinguish between mild and moderate bleeding (overall rate 5.3\%), while the preventive use of a Fogarty balloon in our study was considered a major 'bias' in the interpretation of the results. Anyhow, the fact that we did not experience any grade 2 bleeding does not mean that this is not a common and potentially serious adverse event related to the procedure; it rather suggests that using a prophylactic bronchial blocker is highly recommended in order to reduce and limit such a complication.

Transbronchial Cryobiopsy and Surgical Lung Biopsy
Prolonged postoperative air leaks were much more frequent in the VATS group ( 3.3 vs. $0.3 \%$ ); this result is important, as air leaks lasting longer than 5 days could be associated with an increased rate of empyema [47] and other kinds of pulmonary complications such as atelectasis and pneumonia due to restricted ventilation [48], decreased mobility through chest tubes and related pain, as well as the necessity for pleurodesis, mechanical ventilation, and reoperation [49].

Until now, the identification and careful consideration of preoperative risk factors has been very important to limit the risk of complications associated with SLB for the diagnosis of ILDs. According to the current literature [6, 35], we observed that patients with a more severely impaired percentage predicted of forced vital capacity (FVC\%) have a higher risk of developing adverse events after SLB, while complications after cryobiopsy seem to occur independently of lung function values. These results confirm that one of the advantages of cryobiopsy in the future might be that even patients not able to undergo VATS for functional reasons could be further diagnosed by cryobiopsy.

Although these overall results suggest a much better safety profile of TBLC compared to SLB, the DY of this novel technique should be at least comparable to that of the gold standard in order to be really considered a valid competitor. Due to the lack of studies directly comparing the two procedures, it is difficult to properly decide on this matter. However, our study and the pooled estimate from the literature showed a good DY independently of the criteria used for defining the final diagnosis and of the methods employed for sedation and airway control. Moreover, although a great heterogeneity between studies was observed in terms of design, sample size, and procedural aspects, the DY was $\geq 0.70$ in most of the investigations. This is limited, however, considering the adverse events/mortality with TBLC and SLB taken separately, since in clinical practice the two procedures are not really used competitively; in fact, a patient might undergo TBLC and subsequently VATS if the first approach is not diagnostic. In this specific case, the overall mortality with SLB (3.6\% according to the most recent review of the literature; $95 \% \mathrm{CI}$ $2.1-5.5)$ [36] should not be applied to all patients (100\%) but only to patients in whom cryobiopsy was not diagnostic and who are therefore candidates for VATS (overall cryobiopsy DY $81 \%$ according to our meta-analysis; $95 \%$ CI 75-87). Thereafter, we have two possible scenarios: (1) in the worse scenario, $25 \%$ of the patients undergo SLB because of a previous nondiagnostic cryobiopsy (higher mortality of 5.5\%), and (2) in the better scenario, only $13 \%$ 
of the patients undergo SLB because of a previous nondiagnostic cryobiopsy (lower mortality of 2.1\%) (fig. 7). The 'final' risk of mortality, considering TBLC and VATS together, proves to be $0.3-1.4 \%$, which is significantly lower than the overall mortality with VATS alone. Lastly, we acknowledge some limitations due to the fact that this is a retrospective and single-center study.

In conclusion, TBLC seems to be a safe procedure, with lower complication and mortality rates compared to
SLB. TBLC might, therefore, be considered the first diagnostic approach for obtaining tissue in DPLDs, reserving the surgical approach for only the minority of cases in which TBLC is not adequate or not diagnostic.

\section{Financial Disclosure and Conflicts of Interest}

None of the authors have any conflicts of interest.

\section{References}

$\checkmark 1$ Demedts M, Costabel U: ATS/ERS international multidisciplinary consensus classification of the idiopathic interstitial pneumonias. Eur Respir J 2002;19:794-796.

$\checkmark 2$ Raghu G, Collard HR, Egan JJ, et al: An official ATS/ERS/JRS/ALAT statement: idiopathic pulmonary fibrosis: evidence-based guidelines for diagnosis and management. Am J Respir Crit Care Med 2011;183:788824.

3 Kreider ME, Hansen-Flaschen J, Ahmad NN, Rossman MD, Kaiser LR, Kucharczuk JC, Shrager JB: Complications of video-assisted thoracoscopic lung biopsy in patients with interstitial lung disease. Ann Thorac Surg 2007; 83:1140-1145.

4 Kondoh Y, Taniguchi H, Kitaichi M, Yokoi T, Johkoh T, Oishi T, Kimura T, Nishiyama O, Kato K, du Bois RM: Acute exacerbation of interstitial pneumonia following surgical lung biopsy. Respir Med 2006;100:1753-1759.

5 Utz JP, Ryu JH, Douglas WW, Hartman TE, Tazelaar HD, Myers JL, Allen MS, Schroeder DR: High short-term mortality following lung biopsy for usual interstitial pneumonia. Eur Respir J 2001;17:175-179.

-6 Park JH, Kim DK, Kim DS, Koh Y, Lee SD, Kim WS, Kim WD, Park SI: Mortality and risk factors for surgical lung biopsy in patients with idiopathic interstitial pneumonia. Eur J Cardiothorac Surg 2007;31:1115-1119.

-7 Babiak A, Hetzel J, Krishna G, Fritz P, Moeller P, Balli T, Hetzel M: Transbronchial cryobiopsy: a new tool for lung biopsies. Respiration 2009;78:203-208.

-8 Yarmus L, Akulian J, Gilber CH, Illei P, Shah P, Merlo C, Orens J, Feller-Kopman D: Cryoprobe transbronchial lung biopsy in patients after lung transplantation: a pilot safety chest study. Chest 2013;143:621-626.

-9 Pajares V, Torrego A, Puzo C, Lerma E, Gil De Bernabé MA, Franquet T: Transbronchial lung biopsy using cryoprobes (in Spanish). Arch Bronconeumol 2010;46:111-115.

10 Casoni GL, Tomassetti S, Cavazza A, Colby TV, Dubini A, Ryu JH, Carretta E, Tantalocco P, Piciucchi S, Ravaglia C, Gurioli C, Romagnoli M, Gurioli C, Chilosi M, Poletti V: Trans- bronchial lung cryobiopsy in the diagnosis of fibrotic interstitial lung diseases. PLoS One 2014;9:e86716.

-11 Travis WD, Costabel U, Hansell DM, King TE Jr, Lynch DA, Nicholson AG, Ryerson CJ, Ryu JH, Selman M, Wells AU, et al: An Official American Thoracic Society/European Respiratory Society Statement: update of the international multidisciplinary classification of the idiopathic interstitial pneumonias. Am J Respir Crit Care Med 2013;188:733-748.

12 Raghu G, Collard HR, Egan JJ, Martinez FJ, Behr J, Brown KK, Colby TV, Cordier JF, Flaherty KR, Lasky JA, et al; ATS/ERS/JRS/ ALAT Committee on Idiopathic Pulmonary Fibrosis: An official ATS/ERS/JRS/ALAT statement: idiopathic pulmonary fibrosis: evidence-based guidelines for diagnosis and management. Am J Respir Crit Care Med 2011;183:788-824.

13 Ernst A, Eberhardt R, Wahidi M, Becker HD Herth FJ: Effect of routine clopidogrel use on bleeding complications after transbronchial biopsy in humans. Chest 2006;129:734-737.

14 Singhal S, Ferraris VA, Bridges CR, Clough ER, Mitchell JD, Fernando HC, Shrager JB: Management of alveolar air leaks after pulmonary resection. Ann Thorac Surg 2010;89: 1327-1335.

15 Mueller MR, Marzluf BA: The anticipation and management of air leaks and residual spaces post lung resection. J Thorac Dis 2014; 6:271-284.

-16 Stroup DF, Berlin JA, Morton SC, Olkin I, Williamson GD, Rennie D, Moher D, Becker BJ, Sipe TA, Thacker SB: Meta-analysis of observational studies in epidemiology: a proposal for reporting. Meta-Analysis of Observational Studies in Epidemiology (MOOSE) group. JAMA 2000;283:2008-2012.

17 Nyaga VN, Arbyn M, Aerts M: Metaprop: a Stata command to perform meta-analysis of binomial data. Arch Public Health 2014;72: 39.

18 Greenland S: Variance estimators for attributable fraction estimates consistent in both large strata and sparse data. Stat Med 1987;6: 701-708. Erratum in Stat Med 1994;13:99.
19 Hagmeyer L, Theegarten D, Wohlschläger J, Treml M, Matthes S, Priegnitz C, Randerath WJ: The role of transbronchial cryobiopsy and surgical lung biopsy in the diagnostic algorithm of interstitial lung disease. Clin Respir J 2015, Epub ahead of print.

20 Kropski JA, Pritchett JM, Mason WR, Sivarajan L, Gleaves LA, Johnson JE, Lancaster LH, Lawson WE, Blackwell TS, Steele MP, Loyd JE, Rickman OB: Bronchoscopic cryobiopsy for the diagnosis of diffuse parenchymal lung disease. PLoS One 2013;8:e78674.

21 Pajares V, Puzo C, Castillo D, Lerma E, Montero MA, Ramos-Barbón D, Amor-Carro O, Gil De Bernabé A, Franquet T, Plaza V, Hetzel J, Sanchis J, Torrego A: Diagnostic yield of transbronchial cryobiopsy in interstitial lung disease: a randomized trial. Respirology 2014; 19:900-906.

22 Griff S, Ammenwerth W, Schönfeld N, Bauer TT, Mairinger T, Blum TG, Kollmeier J, Grüning W: Morphometrical analysis of transbronchial cryobiopsies. Diagn Pathol 2011;6:53.

23 Griff S, Schönfeld N, Ammenwerth W, Blum TG, Grah C, Bauer TT, Grüning W, Mairinger T, Wurps H: Diagnostic yield of transbronchial cryobiopsy in non-neoplastic lung disease: a retrospective case series. BMC Pulm Med 2014;14:171.

24 Hernández-González F, Lucena CM, Ramírez J, Sánchez M, Jimenez MJ, Xaubet A, Sellares J, Agustí C: Cryobiopsy in the diagnosis of diffuse interstitial lung disease: yield and costeffectiveness analysis (in English, Spanish). Arch Bronconeumol 2015;6:261-267.

25 O’Donovan JP, Khan KA, Burke L, Murphy DM, Plant BP, Henry MT, Kennedy MP: Bronchoscopic cryobiopsy: initial experience in an interstitial lung disease centre. Irish J Med Sci 2014;183:11(suppl 1):S515S516.

26 Azcuna H, Izquierdo JP, Aburto M, Barredo I, Echeverria JJ, Tena L, Dorado S, Loizaga AG, Romani A, Aramburu A, Uranga A, Esteban C, Capelastegui A: Cryobiopsy in the diagnosis of interstitial lung disease (meeting abstract). Chest 2014;145:3. 
$\longrightarrow 27$ Gershman E, Fruchter O, Benjamin F, Nader AR, Rosengarten D, Rusanov V, Fridel L, Kramer MR: Safety of cryo-transbronchial biopsy in diffuse lung diseases: analysis of three hundred cases. Respiration 2015;90:40-46.

28 Martín SH, Rodríguez VL, Zulet AAI, Rodrigo JAC, Yagüe A, Rivas PC, Mitjans JB, De Heredia JHP: Transbronchial lung biopsy using cryoprobes: results obtained in our hospital. Eur Respir J 2014;44(suppl 58):P685.

29 Pourabdollah M, Shamaei M, Karimi S, Karimi M, Kiani A, Jabbari HR: Transbronchial lung biopsy: the pathologist's point of view. Clin Respir J 2014, Epub ahead of print.

30 Oezkan F, Cortadellas MC, Freitag L, Wessendorf T, Hager T, Darwichez K: Transbronchial cryobiopsies in rigid bronchoscopy - a review of diagnostic yield, times and complications. 3rd European Congress for Bronchology and Interventional Pulmonology, 23-25 April, 2015, Barcelona.

- 31 Fruchter O, Fridel L, Rosengarten D, Rahman NA, Kramer MR: Transbronchial cryobiopsy in immunocompromised patients with pulmonary infiltrates: a pilot study. Lung 2013; 91:619-624.

32 Fruchter O, Fridel L, El Raouf BA, AbdelRahman N, Rosengarten D, Kramer MR: Histological diagnosis of interstitial lung diseases by cryo-transbronchial biopsy. Respirology 2014; 19:683-638.

33 American Thoracic Society; European Respiratory Society: American Thoracic Society/ European Respiratory Society International Multidisciplinary Consensus Classification of the Idiopathic Interstitial Pneumonias. Am J Respir Crit Care Med 2002;165:277-304.

34 Tomassetti S, Piciucchi S, Tantalocco P, Dubini A, Poletti V: The multidisciplinary ap- proach in the diagnosis of idiopathic pulmonary fibrosis: a patient case-based review. Eur Respir Rev 2015;24:69-77.

35 Nguyen W, Meyer KC: Surgical lung biopsy for the diagnosis of interstitial lung disease: a review of the literature and recommendations for optimizing safety and efficacy. Sarcoidosis Vasc Diffuse Lung Dis 2013;30:3-16.

36 Han Q, Luo Q, Xie JX, Wu LL, Liao LY, Zhang XX, Chen RC: Diagnostic yield and postoperative mortality associated with surgical lung biopsy for evaluation of interstitial lung diseases: a systematic review and meta-analysis. J Thorac Cardiovasc Surg 2015;149:13941401.

37 Krasna MJ, White CS, Aisner SC, Templeton PA, McLaughlin JS: The role of thoracoscopy in the diagnosis of interstitial lung disease. Ann Thorac Surg 1995;59:348-351.

38 Bentzon N, Adamsen S, Jacobsen B, Eriksen C, Hansen OH: Videothoracoscopic lung biopsy by a stapling technique. Eur J Surg 1994; 160:543-546.

39 Moore DJ, McParland CP, Bullock MJ, Cartier Y, Hernandez P: Video-assisted thoracoscopic lung biopsy as a possible cause of acute interstitial pneumonia in a patient with nonspecific interstitial pneumonia. Can Respir J 2004;11:437-440.

40 Tian XL, XuWB, Shi JH, et al: Acute exacerbation of usual interstitial pneumonia and nonspecific interstitial pneumonia: analysis of 6 cases (in Chinese). Zhonghua Jie $\mathrm{He} \mathrm{He} \mathrm{Hu} \mathrm{Xi}$ Za Zhi 2008;31:255-259.

41 Corte TJ, Collard H, Wells AU: Idiopathic interstitial pneumonias in 2015: a new era. Respirology 2015;20:697-698.

42 Margaritopoulos GA, Wells AU: The role of transbronchial biopsy in the diagnosis of dif- fuse parenchymal lung diseases: con (in English, Portuguese). Rev Port Pneumol 2012;18: 61-63.

43 Rena O, Casadio C, Leo F: Videothoracoscopic lung biopsy in the diagnosis of interstitial lung disease. Eur J Cardiothorac Surg 1999; 16:624-627.

44 Tomassetti S, Wells AU, Costabel U, Cavazza A, Colby TV, Rossi G, Sverzellati N, Carloni A, Carretta E, Buccioli M, Tantalocco P, Ravaglia C, Gurioli C, Dubini A, Piciucchi S, Ryu JH, Poletti V: Bronchoscopic lung cryobiopsy increases diagnostic confidence in the multidisciplinary diagnosis of idiopathic pulmonary fibrosis. Am J Respir Crit Care Med 2015, Epub ahead of print.

45 Plönes T, Osei-Agyemang T, Elze M, et al: Morbidity and mortality in patients with usual interstitial pneumonia (UIP) pattern undergoing surgery for lung biopsy. Respir Med 2013;107:629-632.

46 Matsuoka K, Kuroda A, Kang A, et al: Surgical results of video-assisted thoracic surgery and risk factors for prolonged hospitalization for secondary pneumothorax in elderly patients. Ann Thorac Cardiovasc Surg 2013;19:18-23.

47 Brunelli A, Xiume F, Al Refai M, Salati M Marasco R, Sabbatini A: Air leaks after lobectomy increase the risk of empyema but not of cardiopulmonary complications: a casematched analysis. Chest 2006;130:1150-1156.

48 Varela G, Jiménez MF, Novoa N, Aranda JL: Estimating hospital costs attributable to prolonged air leak in pulmonary lobectomy. Eur J Cardiothorac Surg 2005;27:329-333.

49 Ochroch A, Barnett R: Synthetic Sealants for Preventing Air Leaks after Pulmonary Resection. Official Newsletter of the Society of Cardiovascular Anesthesiologist, October 2002. 